\section{Dreaded complications of mistaken identity - Hygroma vs effusion following decompressive craniotomy}

\author{
Sir,
}

The first principle of ethical medical practice is primum non nocera (first, do no harm). However, ignorance may sometimes lead to such complications, which could be avoided. Development of subdural hygroma following a decompressive craniectomy though described in literature is infrequently concentrated upon. Development of hygroma is usually considered benign, usually resolving following the replacement of the bone flap due to restoration of normal cerebrospinal fluid flow dynamics. A radiological mimic of this hygroma is effusion, with an incidence of upto $33 \%$, has unfortunately not received much attention in the contemporary literature. We, herein, describe our inexperience in recognizing this entity in a 30 year male patient, who following a decompressive craniectomy came back for replacement of bone flap, had swelling of the scalp flap which on computed tomographic scans showed hypodense collection beneath the meningo-galeal complex, which was misdiagnosed as hygroma. The tormentous postoperative period and morbidity the patient suffered is described in for others to learn from our mistake.

A 30-year-male patient who had undergone right fronto-temporo-parietal decompressive craniectomy for traumatic right fronto-temporo-parietal acute subdural hematoma with parietal contusion and mass effect and midline shift, operated in 2008, presented for replacement of bone flap in 2012. At the time of discharge the patient was having a sensorium of E4M6V5. The patient presented with the same sensorium for bone flap replacement. CT scan of the brain showed hypodense collection beneath the meningo-galeal complex, which was diagnosed as subdural hygroma [Figure 1a]. A lumbar drain was placed in the immediate preoperative period. The flap had sunken to the level with the adjacent skull but had not created a crater. The bone flap was replaced back. In the immediate postoperative period the patient was noticed to have right hemiplegia of grade $2 / 5$. Computed tomography of brain showed persistence of subdural hypodensity with small hyperdense collection at the posterior margin, squashing of lateral ventricles, midline shift and mass effect [Figure 1b]. The patient was immediately taken for removal of bone flap, and evacuation of the loculated fluid. Following the removal of the bone flap, on attempted durotomy, we observed that the dura was abnormally thickened [Figure 1c] and measured approximately half-a-centimeter thick. There was a thin, dirty grey membrane covering the brain surface containing xanthochromic fluid. The membrane was excised, xanthochromic fluid evacuated and a lax duroplasty performed using fascia lata graft. The histopathological examination of the material showed fragments of granulation tissue comprising multiple proliferating blood vessels lined by endothelial cells, fibroblastic proliferation, extravasated red blood cells and mild inflammatory infiltrate and the membrane was diagnosed as chronic subdural membrane. The patient had a tormentous post-operative period with infection of the scalp flap. He started showing some improvement in his limb power after 2-weeks time and at discharge after 4 weeks was able to walk with a spastic hemiplegic gait, without support. He had a power of $4 / 5$ in the upper limbs, distal worser than proximal, requiring help for feeding.

Subdural hygromas akin to hematomas are collections in the subdural space but contain cerebrospinal fluid as opposed to blood clots of various ages..$^{[1-4]} \mathrm{Head}$ injuries, post-decompressive craniotomy, brain atrophy, spinal drainage are few causes of formation of subdural hygroma. ${ }^{[5]}$ Various theories have been described trying to eludicate the natural history of what was proposed by Feng et al., ${ }^{[6]}$ as two ends of the same spectrum. Arachnoid though tightly attached to pial surface by trabeculae is separated by the cushion of cerebrospinal fluid. Arachnoid barrier cell layer is separated from the dura with an interface layer. The meningeal and periosteal dural layers are tightly bound by collagen whereas the innermost dural border cell layer is devoid of collagenous architecture. Any insult to dural arachnoid interface, results in formation of potential space with subsequent formation of subdural hygroma. Added to this the loss of blood brain barrier following trauma leads to increased vascular permeability and hence oncotic pressure in the subdural space thus starting a vicious cycle. ${ }^{[7-9]}$ Arachnoidal injury leads 


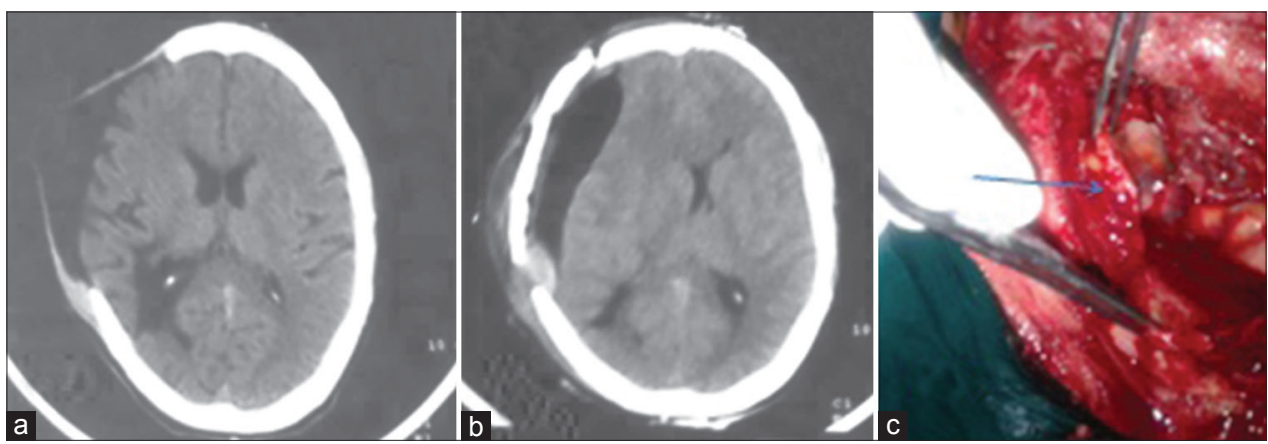

Figure 1: (a) CT scan of brain showing the post-decompressive status with hypodense collection beneath the meningo-galeal complex with no evidence of mass effect. (b) Post-bone flap replacement CT scan showing mild extradural hyperdense collection, with hypodense subdural collection with midline shift and mass effect. (c) Intraoperative picture showing thickened dural membrane (arrow)

to leakage of cerebrospinal fluid with a ball-valve effect. The fluid so collected, remains unabsorbed and thus the vicious cycle and fluid stagnation. Subdural hygromas with gradual detachment from circulation leads to increase in protein content, triggering of inflammatory response, neomembrane formation with fragile capillaries.

Altered cerebrospinal fluid dynamics and post decompressive craniectomy sequel can be prevented by augmented duraplasty which may restore cerebrospinal fluid dynamics and prevent the formation of subdural hygroma. ${ }^{[5]}$

On computed tomography, hygromas are hypodense whereas subdural hematomas vary from hypo to hyperdense depending upon their chronological age (hyperacute- isodense; acute- hyperdense due to clot retraction; subacute- iso to hypodense due to degradation of proteins in the clot; chronic- hypodense due to liquefaction of clot). ${ }^{[10]}$ Signal intensities in Magnetic resonance imaging for hygromas are identical to CSF in all pulse sequences whereas they vary in intensities in subdural hematomas depending upon the age and pulse sequences (hyperactue stage: T1- iso, T2-hyper; acute stage: T1-iso, T2- hypo; subacute: T1 and T2- hyper; chronic- T1 and T2- hypo). ${ }^{[11]}$ Diffusion-weighted images will, however, show restricted diffusion in cases of subdural hematoma. However, to obtain MR imaging requires a strong suspicion. The protein content in hygromas is identical to that of CSF whereas effusions have high protein contents with relatively normal sugar levels, thus differentiating them from fluid collections secondary to infections. ${ }^{[12]}$

Hygromas are rarely symptomatic and most of them resolve spontaneously. However, symptomatic cases require surgical intervention wherein replacement of bone flap is considered the best option. However, caution has to be exercised, to monitor features of raised intracranial pressure. In case of raised intracranial pressure, the collection has to be drained off, either pre- or intraoperatively. ${ }^{[12]}$

A repeat imaging study to know the change in the subdural collection, before replacement of bone flap might be useful.

Omekareswar Rambarki, Alugolu Rajesh Department of Neurosurgery, Nizam's Institute of Medical Sciences, Punjagutta, Hyderabad, India

Address for correspondence: Dr. A. Rajesh, Department of Neurosurgery, Nizam's Institute of Medical Sciences, Punjagutta, Hyderabad - 500 082, India. E-mail: drarajesh1306@gmail.com

\section{References}

1. Schachenmayr W, Friede RL. The origin of subdural neomembranes. I. Fine structure of the dura-arachnoid interface in man. Am J Pathol 1978;92:53-68.

2. Haines DE. On the question of a subdural space. Anatomical Record 1991;230:21.

3. Haines DE, Harkey HL, Al-Mefty O. The 'subdural' space: A new look at an outdated concept. Neurosurgery 1993;32:111-20.

4. Orlin JR, Osen KK, Hovig T. Subdural compartment in pig: A morphologic study with blood and horseradish peroxidase infused subdurally. Anat Rec 1991;230:22-37.

5. Yang XF, Wen L, Shen F, Li G, Lou R, Liu WG, et al. Surgical complications secondary to decompressive craniectomy in patients with a head injury: A series of 108 consecutive cases. Acta Neurochir (Wien) 2008;150:1241-7.

6. Feng JF, Jiang JY, Bao YH, Liang YM, Pan YH. Traumatic subdural effusion evolves into chronic subdural hematoma: Two stages of the same inflammatory reaction? Med Hypotheses 2008;70:1147-9.

7. Lee KS. The pathogenesis and clinical significance of traumatic subdural hygroma. Brain Inj 1998;12:595-603.

8. Jeon SW, Choi JH, Jang TW, Moon SM, Hwang HS, Jeong JH. Risk factors associated with subdural hygroma after decompressivecraniectomy in patients with traumatic brain injury: A comparative study. J Korean Neurosurg Soc 2011;49:355-8.

9. Aarabi B, Chesler D, Maulucci C, Blacklock T, Alexander M. Dynamics of subdural hygroma following decompressive craniectomy: A comparative study. Neurosurg Focus 2009;26:E8. 
10. Lee KS, Bae WK, Bae HG, Yun IG. The fate of traumatic subdural hygroma in serial computed tomographic scans. J Korean Med Sci 2000;15:560-8.

11. Fobben ES, Grossman RI, Atlas SW, Hackney DB, Goldberg HI, Zimmerman RA, et al. Characteristics of subdural hematomas and hygromas at 1.5 T. AJR Am J Roentgenol 1989;153:589-95.

12. Paredes I, Cicuendez M, Delgado MA, Martinez-Pérez R, Munarriz PM, Lagares A. Normal pressure subdural hygroma with mass effect as a complication of decompressive craniectomy. Surg Neurol Int 2011;2:88.

\begin{tabular}{|l|l|}
\hline \multicolumn{2}{|c|}{ Access this article online } \\
\hline Quick Response Code: & Website: \\
\hline & www.ruralneuropractice.com \\
\cline { 2 - 3 } & \\
\hline
\end{tabular}

